\title{
Taylor's Expansion in a Distribution Algebra
}

L. BERG

Für eine gewisse Distributionenalgebra wird gezeigt, daß jedes Element der Algebra einen Wert besitzt sowie durch seine 'Taylorentwicklung dargestellt wird. Außerdem wird die Struktur der Teilalgebra der. Wérte untersucht.

Для, некоторой дистриоуциониой алгебры показывается, что каждыli элемент алгебры имеет значение и представим разложением. Тейлора. Кроме того нсследуетсн структура подалгебры значений.

For a certain distribution algebra it is shown that every element of the algebra possesses a value and is representable by its Taylor's expansion. Moreover there is investigated the structure of the subalgebra of values.

By definition, a distribution algebra is an associative, but noncommutative differential algebra with at least one element $h$ satisfying

$$
h^{2} \doteq h
$$

and $h^{\prime} \neq 0$. A good survey on some clissses of distribution algebras with additional properties was given by MA KYrN MYrNT [3]. Here we go back to the more general distribution algebras of [1].

The aim of this paper is to show that every element of the latter distribution algebras is representable by its Taylor's expansion, using the notion of values of such elements introduced in [2]. As a consequence we are able to determine the structure of the set of these values.

\section{Preliminaries}

In [1] there was considered the distribution algebra $D_{1}$ with unit element 1 generated by two elements $t, h$ with $\iota_{\ell}$ the properties

$$
t^{\prime}=1, \cdots \quad t h^{\prime}=0
$$

and, of course, (1). The ring of scalars of this algebra is assumed to contain the rational numbers. According to Dirac the derivative $h^{\prime}$ is denoted by $\delta$, the more as this element shall be interpreted as a Schwartz distribution. As a consequence of (2), in $D$. there are valid the well known relations

$$
\iota^{n} \delta^{(m)}=(-1)^{n} n !\left(\begin{array}{l}
m \\
n
\end{array}\right) \delta^{(m-n)}
$$

for all integers $m, n \geqq 0$ with $\left(\begin{array}{l}m \\ n\end{array}\right)=0$ for $m<n$. As a consequence of (1), in $D_{1}$ 
there are valid the relations

$$
h^{(m)}=\sum_{n=0}^{m}\left(\begin{array}{c}
m \\
n
\end{array}\right) h^{(n)} \cdot h^{(m-n)},
$$

too, where the products on the right-hand side are no Schwartz distributions for $m>1$.

In [1] there was also constructed the extension $D_{2}$ of $D_{1}$ as the distribution algebra of the elements

$$
f=\sum_{n=0}^{\infty}\left(a_{n} t^{n}+b_{n} t^{n} h\right)
$$

where $a_{n}, b_{n}$ are polynomials in $\delta^{(k)}(k=0,1,2, \ldots)$, which are determined uniquely by $f$ and can be prescribed arbitrarily. With the elements (5) all operations in $D_{2}$ are to be carried out termwise. In $D_{2}$ two elements $f, g$ are defined to be equal if and only if

$$
\therefore \quad(f-g) \delta^{(m)}=0
$$

for $m=0,1,2, \ldots$ According to this definition a sequence $f_{n}$ is defined to converge to the limit $f \in D_{2}$, if for every fixed $m \geqq 0$ there is

$$
\left(f \circ f_{n}\right) \delta^{(m)}=\mathbf{0}
$$

for suitable large $n$. With these definitions e.g. the equátions

$$
t^{i} h t^{k}=t^{i+k} h+k \sum_{j=1}^{\infty} \frac{(-1)^{j-1}}{(i+j)(i+k+j)(j-1) !} \delta^{(i-1)} t^{i+k+j}
$$

are valid in $D_{2}$.

\section{Series}

In what follows we need series with more general coefficients than in (5). For this case we consider the

Theorem 1: For an arbitrary sequence $f_{k}$ of elements from $D_{2}$ the series

$$
f=\sum_{k=0}^{\infty} f_{k} t^{k}
$$

is always converging to an element from $D_{2}$.

Proof: According to (5) all elements $f_{k}$ possess representations of the form"

$$
f_{k}=\sum_{i=0}^{\infty}\left(a_{k i} i^{i}+b_{k i} i^{i} h\right)
$$

Hence in view of $(6)$ we have

$f_{k} t^{k}=\sum_{i=0}^{\infty}\left(a_{k i} t^{i+k}+b_{k i}\left(t^{i+k} h+k \sum_{j=1}^{\infty} \frac{(-1)^{j-1}}{(i+j)(i+k+j) \cdot(j-1) !} \delta^{(i-1) t^{i+k+j}}\right)\right)$.

Summing over $k$ and substituting $i+k=n$ and $i+k+j=n$, respectively, we find that the element $f$ from (7) possesses also the form (5) with

$$
a_{n}=\sum_{i=0}^{n}\left(\dot{a}_{n-i, i}+\sum_{j=1}^{n-i} b_{n-i-i, i} \frac{(n-i-j)(-1)^{j-1}}{(i+j) n(j-1) !} \delta^{(j-1)}\right) \text { and } b_{n}=\sum_{i=0}^{n} b_{n-i, i}
$$


i.e. (7) is an element from $D_{2 .}$. All foregoing calculations with infinite series are possible in view of the definition of convergenee, because all series terminate after multiplication by $\delta^{(m)}$, so that in fact no convergence problems arise

\section{Values}

In [2] there were considered the series

$$
f_{0}=\sum_{k=0}^{\infty} \frac{(-1)^{k}}{k !} f^{(k)} t^{k}
$$

for some elements $f$ of $D_{2}^{\prime}$. Since by Taylor's theorem for holomorphic functions $f(t)$ and small numbers $t$ the series (8) is equal to the value $f(0)$, the element $f_{0}$ from (8) is named the (generalized) vilue of $f$ (at the point $t=0$ ). In [2] there was already proved that $t_{0}=0$ and $\left(t^{n} h\right)_{0}=0$ for all natural numbers $n$,

$$
\begin{aligned}
& h_{0}^{2}=h_{0}, \quad h_{0} t=t h_{0} ; \quad \delta_{0} t=t \delta_{0}, \\
& \delta_{0}^{(m)} t-t \delta_{0}^{(m)}=m \delta_{0}^{(m-1)}
\end{aligned}
$$

for $m \geqq 1$ as well as

$$
\begin{aligned}
& \delta^{(n)} \delta^{(m)}=\sum_{i=0}^{m}(-1)^{i}\left(\begin{array}{c}
m \\
i
\end{array}\right) \delta_{0}^{(n+i)} \delta^{(m-i)} \\
& \left(\delta^{(n)} \delta^{(m)}\right)_{0}=\sum_{i=0}^{m}(-1)^{i}\left(\begin{array}{c}
m \\
i
\end{array}\right) \delta_{0}^{(n+i)} \delta_{0}^{(m-i)}
\end{aligned}
$$

or $m, n \geqq 0$ and

$$
\left(f_{0}{ }^{\prime}\right)=0
$$

for $f \doteq h^{(n)}, n \geqq 0$.

Theorem 2: The value (8) exists for every element $f \in D_{2}^{\prime}$ and has always the property (13): The value-operator is linear, i.e. it satisfies

$$
(\alpha f+\beta g)_{0}=\alpha f_{0}+\beta g_{0}
$$

in the case $\alpha^{\prime}=\beta^{\prime \prime}=0$. Further relations for arbitrary $f, g \in D_{2}$ are

$$
\begin{aligned}
& (f g)_{0}=\sum_{k=0}^{\infty} \frac{(-1)^{k}}{k !} f^{(k)} g_{0} t^{k}, \\
& (f t)_{0}=0, \quad(f h)_{0}=f_{0} h_{0}, \cdots \quad(f \delta)_{0}=f_{0} \delta_{0} .
\end{aligned}
$$

Proof: Since the series in (8) possesses the form (7), Theorem 1 implies the first assertion. The equations (13) and (14) are easily, verified from (8). The equation

$$
\begin{aligned}
(f g)_{0} & =\sum_{i=0}^{\infty} \frac{(-1)^{i}}{i !}(f g)^{(i)} t^{i}=\sum_{i=0}^{\infty} \frac{(-1)^{i}}{i !} \sum_{k=0}^{i}\left(\begin{array}{l}
i \\
k
\end{array}\right) f^{(k)} g^{(i-k)} t^{i} \\
& =\sum_{k=0}^{\infty} \sum_{i=k}^{\infty} \frac{(-1)^{i}}{k !(i-k) !} l^{(k)} g^{(i-k)} t^{i}=\sum_{k=0}^{\infty} \frac{(-1)^{k}}{k !} f^{(k)} \sum_{j=0}^{\infty} \frac{(-1)^{j}}{j !} g^{(j) t^{j+k}}
\end{aligned}
$$

with $j=i-k$ shows in view of (8) with $g$ instead of $f$ that (15) is valid, too: From (15). and $t_{0}=0$ as well as (9) it.follows (16). Hence Theorem 2 is proved 
Of course, both scalar multiples of the unit element 1 and the values thenselves are their own values. These statements and the equations (13) and (14) show that the values (8) behave like ordinary constants in calculus. Equation (12) is a special application of (14) to equation (11). Note that formula (14) is, generally speaking, not valid in the case that the constants are standing at the right of the elements. A general consequence of $(15)$ is the equation $(f g)_{0}=\left(f g_{0}\right)_{0}$.

\section{'Taylör's expansion}

Now we come to another property which-underlines the right of terming the elements (8). values.

Theor em 3: All elements $f \in D_{2}$ are réspresentable by Taylor's expansion

$$
j=\sum_{n=0}^{\infty} \frac{1}{n !} f_{0}^{(n)} t^{n}
$$

and they sutisfy for arbitrary integers $m \geqq 0$ the equation

$$
f \delta^{(m)}=\sum_{n=0}^{m}(-1)^{n}\left(\begin{array}{c}
m \\
n
\end{array}\right) f_{0}^{(n)} \delta^{(m-n)} .
$$

Proof: Multiplying (17) by $\delta^{(m)}$ and considering (3) it follows (18). Hence, in view of the definition of equality in $D_{2}$ it suffices to prove (18). Applying (8) to $f^{(n)}$ instead of $f$ we obtain

$$
f_{0}^{(n)}=\sum_{k=0}^{\infty} \frac{(-1)^{k}}{k !} f^{(n+k)} t^{k}
$$

hence, in view of (3)

$$
f_{0}^{(n)} \delta^{(m-n)}=\sum_{k=0}^{m-n}\left(\begin{array}{c}
m-n \\
k
\end{array}\right) f^{(n+k)} \delta^{(m-n-k)}=\sum_{i=n}^{m}\left(\begin{array}{c}
m-n \\
j-n
\end{array}\right) \cdot f^{(i)} \delta^{(m-j)}
$$

with $j=n+k$ and therefore

$$
\sum_{n=0}^{m}(-1)^{n}\left(\begin{array}{c}
m \\
n
\end{array}\right) f_{0}^{(n)} \delta^{(m-n)}=\sum_{j=0}^{m} \sum_{n=0}^{j}(-1)^{n}\left(\begin{array}{c}
m \\
n
\end{array}\right)\left(\begin{array}{c}
m-n \\
j-n
\end{array}\right) f^{(j)} \delta^{(m-j)}
$$

According to $\left(\begin{array}{c}m \\ n\end{array}\right)\left(\begin{array}{c}m-n \\ j \div n\end{array}\right)=\left(\begin{array}{c}m \\ j\end{array}\right)\left(\begin{array}{l}j \\ n\end{array}\right)$ and

$$
\sum_{n=0}^{j}(-1)^{n}\left(\begin{array}{l}
j \\
n
\end{array}\right)=\left\{\begin{array}{lll}
1 & \text { for } & j=0 \\
0 & \text { for } j>0
\end{array}\right.
$$

the foregoing sim is equal to $f \delta^{(m)}$ and Theorem 3 is proved

For holomorphic functions $/$ equation (18) is well known in the theory of Schwartz distributions. Equation (11) is a special case of (18) with $f=\delta^{(n)}$. Equation (18) reads for $m=0$

$$
j \delta=f_{0} \delta \text {. }
$$

Conversely, if

$$
f \delta=c \delta
$$


with $c^{\prime}=0$, then it follows $c=f_{0}$, because (19) and (20) imply $\left(f_{0}-c\right) \delta=0$ and therefore $\left(f_{0}-c\right) \delta^{(n)}=0$ for every $n \geqq 0$, so that we.obtain the assertion $f_{0}=c$. Differentiating equation (19) $n$-times and considering the value of the result we obtain

$$
f_{0} \delta_{0}^{(n)}=(f \delta)_{0}{ }^{(n)}
$$

This equation implies inductively

$$
\left.\begin{array}{l}
f_{0} \delta_{0}{ }^{(m)} \delta_{0}{ }^{(n)}=\left((f \delta)^{(m)} \delta\right)_{0}{ }^{(n)} \\
f_{0} \delta_{0}{ }^{(k)} \delta_{0}{ }^{(m)} \delta_{0}{ }^{(n)}=\left(\left((f \delta)^{(k)} \delta\right)^{(m)} \delta\right)_{0}{ }^{(n)} \\
\ldots \ldots \ldots \ldots \ldots \ldots \ldots \ldots \ldots \ldots \ldots
\end{array}\right\}
$$

It should be possible to use 'Taylor's expansion also to. define values of elements of $D_{2}$ in a point different from $t \stackrel{\doteq}{=} 0$, however, up to now this is not worked out.

\section{An isomorphism -}

The following theorem clarifies the structure of the set of values.

Theorem 4: The values (8) form a subalgebra $A$ of $D_{2}$, which is generated by the special values 1 and $h_{0}^{(n)}(n \geqq 0)$, and which is isomorphic to the subalgebra $D_{0}$ generated by the elements 1 and $h^{(n)}(n \geqq 0)$. The isomorphism $D_{0} \rightarrow A$ is generated by the mapping

$$
\text { '1 } \rightarrow 1 ; \quad h^{(n)} \rightarrow n ! h_{0}^{(n)}
$$

Proof: From $(5),(16)$ and $t_{0}=0$ we find

$$
f_{0}=a_{00}+b_{00} h_{0}
$$

(the first zero is the index $n=0$ from (5), the second zero indicates the value operation). Since (14) and (18) imply

$$
\left(f \delta^{(m)}\right)_{0}=\sum_{n=0}^{m}(-1)^{n}\left(\begin{array}{l}
m \\
n
\end{array}\right) f_{0}^{(n)} \delta_{0}^{(m-n)}
$$

and $a_{0}, \bar{b}_{0}$ are polynomials in $\delta^{(k)}$, the values $a_{00}$ and $b_{00}$ are polynomials in $\delta_{0}{ }^{(k)}$. Hence, (24) implies that all values $f_{0}$ are contained in the algebra $A$ generated by $h_{0}{ }^{(n)}$ :

Next we derive from (4)

$$
h_{0}^{(m)}=\left(h h^{(m)}\right)_{0}+\sum_{n=1}^{m-1}\left(\begin{array}{c}
\dot{m} \\
\dot{n}
\end{array}\right)\left(h^{(n)} h^{(m-n)}\right)_{0}+\left(h^{(m)} h\right)_{0} .
$$

Considering $\delta=h^{\prime}$, equation (12) can be written in the form

$$
\cdot\left(h^{(n)} h^{(m)}\right)_{0}=\sum_{i=0}^{m-1}(-1)^{i}\left(\begin{array}{c}
m-1 \\
i
\end{array}\right) h_{0}{ }^{(n+i)} h_{0}(m-i)
$$

with $m, n \geqq 1$, so that with $j=n+i$ we have

$$
\begin{aligned}
\sum_{n=1}^{m-1}\left(\begin{array}{l}
m \\
n
\end{array}\right)\left(h^{(n)} h^{(m-n)}\right)_{0} & \left.=\sum_{n=1}^{m-1} \sum_{i=0}^{m-n-1}\left(\begin{array}{c}
m \\
n
\end{array}\right)(-1)^{i}\left(\begin{array}{c}
m-n-1 \\
i
\end{array}\right) h_{0}{ }^{(n+i)} h_{0}{ }^{(m-n-i)}\right) \\
& =\sum_{n=1}^{m-1} \sum_{j=n}^{m-1}(-1)^{n+j}\left(\begin{array}{c}
m^{i} \\
n
\end{array}\right)\left(\begin{array}{c}
m-n-1 \\
j-n
\end{array}\right) h_{0}^{(j)} h_{0}{ }^{(m-j)}
\end{aligned}
$$


According to

$$
\sum_{n=1}^{j}(-1)^{n+j}\left(\begin{array}{c}
m \\
n
\end{array}\right)\left(\begin{array}{c}
m-n-1 \\
j-n
\end{array}\right)=1-\left(\begin{array}{c}
m-1 \\
j
\end{array}\right)(-1)^{j}
$$

this implies

$$
\sum_{n=1}^{m-1}\left(\begin{array}{c}
m \\
n
\end{array}\right)\left(h^{(n)} h^{(m-n)}\right)_{0}=\sum_{j=0}^{m-1}\left(1-\left(\begin{array}{c}
m-1 \\
j
\end{array}\right)(-1)^{j}\right) h_{0}^{(j)} h_{0}^{(m-j)},
$$

and from (18) with $f=h, \delta=h^{\prime},(16)$ and (25) we obtain equation

$$
h_{0 \rightarrow}^{(m)}=\sum_{n=0}^{m} h_{0}^{(n)} h_{0}^{(m-n)}
$$

Obviously, the mapping (23) transfers equation (4) into equation (26). Since $D_{0}$ is nothing else than the free algebra generated by $h_{0}{ }^{(n)}$, which is endowed with the relations (4) (cf. [1]), and since the corresponding relations (26) under the mapping (23) are also valid, $D_{0}$ has at least a homomorphic image in $A$. 'The fact that all elements of $D_{0}^{\prime}$ possess the normal form $a+b h^{\prime}$ with polynomials $a, b$ in $\delta^{(k)}$ implies that all elements of $A$ possess by the mapping (23) the normal form $c+d h_{0}$ with polynomials $c, d$ in $\delta_{0}{ }^{(k)}$. The equations (22) show that the polynomials are always values of polynomials $a_{0}, b_{0}$ in $\delta^{(k)}$. Hence, every element of $A$ is a value of an element of $D_{0}$.

It remains to show that the homomorphism generated by (23) is one-to-one. Assume that this is not the case, i.c. that for an element $a+b h \neq 0$ in $D_{0}$ the image $c+d h_{0}$ in $\dot{A}$ is vanishing. Iret $c=a_{00}, d=b_{00}$ as before. Then according to $\delta=h \delta+\delta h$, (19) and (16) we have

$$
0=\left(a_{00}+b_{00} h_{0}\right)^{\prime} \delta=a_{0} \delta+b_{00} h \delta=\left(a_{0}+b_{0}\right) \delta-b_{0} \delta h
$$

and this implies $b_{0}^{\prime}=a_{0}=0$. The polynomial $c$ has the form

$$
c=\sum \gamma_{m} \delta_{0}^{\prime}\left(k_{m 1}\right) \delta_{0}{ }^{\left(k_{m s}\right)} \ldots \delta_{0}{ }^{\left(k_{m n_{m}}\right)}
$$

so that according to (22)

$$
a_{0}=\sum \gamma_{m}\left(\ldots\left(\delta^{\left(k_{m 1}\right)} \delta\right)^{\left(k_{m 2}\right)} \ldots \delta\right)^{\left(k_{m n_{n}}\right)}=0 .
$$

Considering in this sum the addends with maximal $n_{m}$, from these once more the addends with maximal $k_{m n_{m}}$, from these the addends with maximal $k_{m, n_{m}-1}$ etc., we find an addend, from which the tern

$$
\gamma_{m} \delta^{\left(k_{m 1}\right)} \delta^{\left(k_{m 1}\right)} \ldots \delta^{\left(k_{m n_{m}}^{\prime}\right)}
$$

appearing after evaluating the brackets in (27) cannot be canceled against another term in the sum: Hence, this special $\gamma_{m}$ must vanish, and therefore all $\gamma_{m}$ must vanish. This means that $c$ is the zero-polynomial and so must be $a$. Analogously, $d$ is the zero-. polynomial and $b$, too, but this contradicts the assumption $a+b h \neq 0$. So Theorem 4 is proved

The algebra $D_{0}$ is a distribution algebra, but the algebra $A$ not, though it is in view of (13) a trivial differential algebra. The isomorphism (23) allows to transfer all relations from $D_{0}$ into relations in $A$. For example from $h \delta^{2}=\delta^{2} h, h \delta h=0$ we immediately obtain $h_{0} \delta_{0}{ }^{2}=\delta_{0}{ }^{2} h_{0}, h_{0} \delta_{0} h_{0}=0$. 


\section{REFERENCES}

[1] Berg, L. : Construction of distribution algebras. Math. Nachr. 82 (1978), 255-262.

[2] - : Values in a distribution algebra. Demonstratio Math. 12 (1979), 639-644.

[3] Ma Kyin Mrrst: Distribution Algebra (Mi. Se.-Thesis). Rangoon: Arts and Science University 1979.

Manuskripteingang: 06. 09.1982

VERFASȘER:

Prof. Dr. Lothar Bfrg

Sektion Mathematik der Wilhelm-Pieck-Universität

DDR -2500 Rostock, Universitätsplatz 1 